\title{
Optimum range of angle tracking radars: a theoretical computing
}

\author{
Sadegh Samadi ${ }^{1}$, Mohammad Reza Khosravi², Jafar A. Alzubi ${ }^{3}$, Omar A. Alzubi ${ }^{4}$, Varun G. Menon \\ ${ }^{1,2}$ Shiraz University of Technology, Iran \\ ${ }^{3,4} \mathrm{Al}$-Balqa Applied University, Jordan \\ ${ }^{5}$ SCMS School of Engineering and Technology, India
}

\begin{tabular}{l} 
Article Info \\
\hline Article history: \\
Received Mar 7, 2018 \\
Revised Dec 22, 2018 \\
Accepted Jan 3, 2019 \\
\hline
\end{tabular}

\section{Keywords:}

Angle error

Angle tracking radars (ATRs)

Optimal range

Radar equation

Signal-to-noise ratio (SNR)

\begin{abstract}
In this paper, we determine an optimal range for angle tracking radars (ATRs) based on evaluating the standard deviation of all kinds of errors in a tracking system. In the past, this optimal range has often been computed by the simulation of the total error components; however, we are going to introduce a closed form for this computation which allows us to obtain the optimal range directly. Thus, for this purpose, we firstly solve an optimization problem to achieve the closed form of the optimal range $\left(R_{\text {opt. }}\right)$ and then, we compute it by doing a simple simulation. The results show that both theoretical and simulation-based computations are similar to each other.
\end{abstract}

Copyright $@ 2019$ Institute of Advanced Engineering and Science. All rights reserved.

\section{Corresponding Author:}

Varun G. Menon,

SCMS School of Engineering and Technology,

Kochi, India.

Email: varunmenon@scmsgroup.org

\section{INTRODUCTION}

There are different kinds of radar systems for different applications in industries, airports, military organs such as navy and so on. A type of radars is the tracking radars which are applied in fire control and etc [1]. Generally, two classes of tracking radars can be considered as angle tracking and range tracking radars. Our aim in this study attends to the first class. Figure 1 shows a real sample of tracking radar which has been placed on a ground platform for the fire control usages. Tracking radar for finding angle can use several tracking modes (scanning techniques) including sequential lobing, conical scan and monopulse (e.g. in terms of amplitude) [1]. These three techniques are different in terms of the error status. So, the mode used in each angle tracking radar must be considered in computing the error and then the optimal range of radar. Figure 2 shows a schematic which explains the mechanism of angle tracking radars. From step 1 to step 6, a target is seen in PPI display which tracked during these steps. Figure 3 and Figure 4 show the block diagram of two types of angle tracking radars based on monopulse and conical scan techniques, respectively [2], [3]. In [1]-[3], it is explained that in terms of angle error, two techniques of sequential lobing and conical scan are similar to each other. In the next parts, we specify the differences between the impacts of monopulse and conical scan/sequential lobing (non-monopulse) techniques. Computing the optimal range of each angle tracking radar is an optimization problem which depicts one of the main parameters of the radar. In [1], this problem has been represented, but it is only solved by simulation and numerical computation. As a completing approach for [1], we wish to solve this problem theoretically. In addition, we obtain the simulation result to compare to the proposed approach.

In the appendix of this paper, we give the readers a sample MATLAB code in order to the simulation-based computing of the optimal range. 


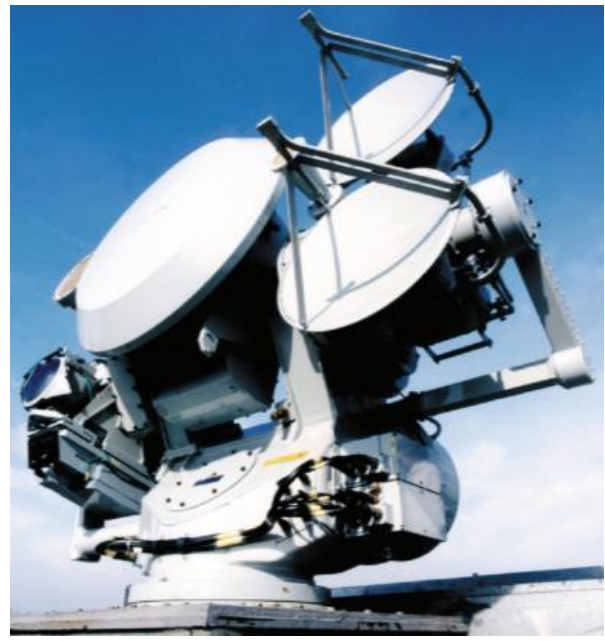

Figure 1. A fire control tracking radar

This paper is organized as follows. In the second part, we have an overview on the resources of the angle error. In the third part, we theoretically solve the problem. In the forth part, we solve a typical example based on both simulation-based and theoretical approaches which allows us to have a comparison between them. The latest parts are allocated to the conclusions of the work and the appendix.
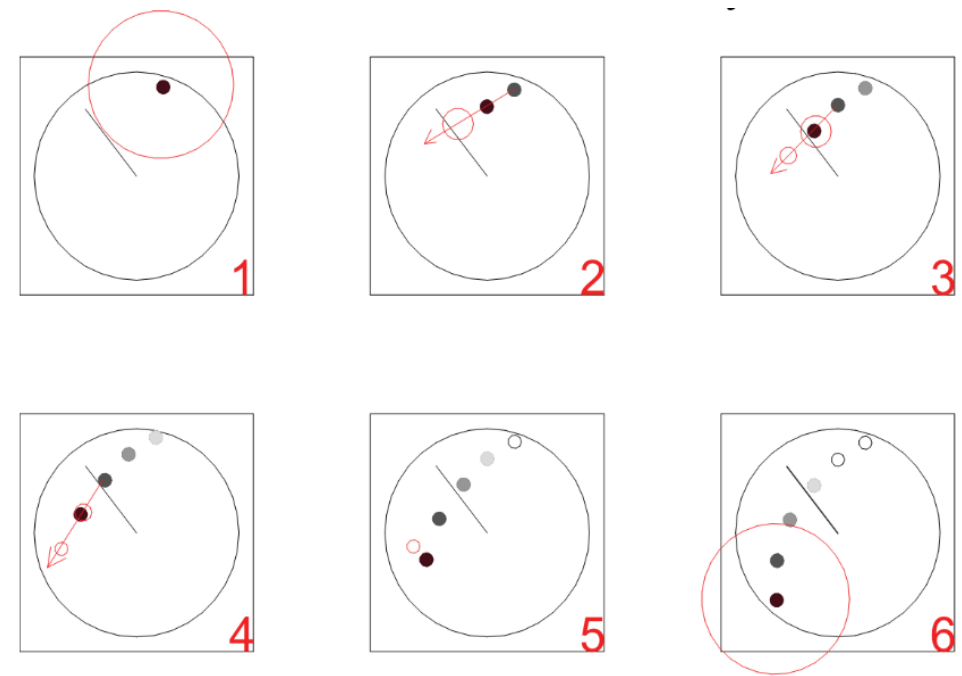

Figure 2. PPI display sequence to illustrate the target tracking process [2]

\section{RESOURCES OF ERROR IN ATRS}

According to [1], the general standard deviation of the angle error $\left(\sigma_{t}\right)$ in ATRs is mainly depended on five components (five different standard deviations) including angle fluctuation error related to angle noise and glint phonomena $\left(\sigma_{g}\right)$, mechanical error $\left(\sigma_{\text {mech. }}\right)$, control loop error $\left(\sigma_{\text {cont. }}\right)$, scintillation error related to amplitude fluctuation and target fading $\left(\sigma_{s c}\right.$. $)$. And finally the error created by thermal noise of the radar system $\left(\sigma_{n}\right)$. As follows, we introduce the detailed information about $\sigma_{t}$. (1) shows the general formula of $\sigma_{t}$.

$$
\sigma_{t}=\sqrt{\sigma_{\text {cont. }}^{2}+\sigma_{\text {mech. }}^{2}+\sigma_{g}^{2}+\sigma_{n}^{2}+\sigma_{\text {sc. }}^{2}}
$$


Where $\sigma_{c o n t .}, \sigma_{\text {mech. }}, \sigma_{g}, \sigma_{n}$ and $\sigma_{s c .}\left(\sigma_{s c}\right.$ is zero for monopulse mode) are as (2) to (6), respectively ((6) is for non-monopulse modes).

$$
\begin{aligned}
& \sigma_{\text {cont. }}=\left\{\begin{array}{lr}
\frac{\omega_{\max }}{k_{v}} & \text { for fixed velocity } \\
\frac{\omega_{\max }}{k_{a}} & \text { for fixed acceleration }
\end{array}\right. \\
& \sigma_{\text {mech. }}=q_{1} \theta_{B} \\
& \text { where } q_{1} \in[0.001,0.005] ; q_{1, \text { typical }}=0.002 \\
& \sigma_{g}=\frac{L}{3 R} \\
& \sigma_{n}=\frac{\theta_{B}}{q_{2} \sqrt{(2 \times S N R)\left(\frac{P R F}{B_{s}}\right)}} \\
& \text { where } q_{2}=\left\{\begin{array}{rr}
1.3 & \text { non-monopulse } \\
1.7 & \text { monopulse }
\end{array}\right. \\
& \sigma_{s c .}=0.01 \theta_{B}
\end{aligned}
$$

In the equations, $B_{s}, k_{v}$ and $k_{a}$ are some constants related to the status of movement of the target and depended on transform function of the control loop, see more information in [4]. $\omega_{\max }$ is the highest angle frequency of the target. $\theta_{B}$ is the maximum of beam width in two directions of elevation and azimuth $\left(\theta_{B}{ }^{\circ}=\max \left\{\theta_{a z}{ }^{\circ}, \theta_{e l}{ }^{\circ}\right\}\right) . L$ is the largest size of the target and $R$ is the distance between radar and target. SNR is signal-to-noise ratio of radar while receiving an echo from the target. $S N R$ is based on the radar equation; we will discuss more about it later. $P R F$ shows the pulse repetition frequency which is equal to the inverse of pulse repetition interval (shown by $T_{p}$ ) in pulse radar-based tracking systems. About SNR and radar equation, we can say that $S N R$ is desired at the input side of the receiver and is therefore computable by the radar equation as (7); for simplicity, we omit some details of radar equation which are reachable in [1], [4].

$$
S N R=\frac{P_{t} \lambda^{2} G_{a}^{2} R C S}{(4 \pi)^{3} P_{\text {noise }} R^{4}}
$$

Where $P_{t}$ the maximum of transmission power of the radar is, $\lambda$ is the wavelength used in the radar. $G_{a}$ is antenna gain of the radar (assume that we use a monostatic pulse radar for angle tracking) and described by (8) where $G_{o}=32000$, and $L_{e l}$ and $L_{a z}$ are the size of the radar's antenna for elevation and azimuth, respectively, see more details in [1]. RCS shows the radar cross section of target and is not often fixed, especially in fast maneuvering and high rate fluctuation, however it is usually replaced with an average value which is at most equal to the geometrical cross section of the target (target size). So in here, we suppose that it is a constant. Finally, $P_{n o i s e}$ is power of the thermal noise of radar's receiver which is computed by (9); in this equation $B$ is the noise bandwidth of the radar's receiver in which $B=\tau^{-1}$ (where $\tau$ is pulse width), $F$ is the noise figure (NF), $k$ and $T_{0}$ are two constants; for more information in this regard, see [1].

$$
\begin{aligned}
& G_{a}=\frac{G_{o} L_{e l} L_{a z}}{(60 \lambda)^{2}} \\
& P_{\text {noise }}=k T_{\circ} B F
\end{aligned}
$$


In addition, there is another form for representing the noise power $P_{\text {noise }}$, refer to [1]. It is observable that in a specific example, all values of $P_{t}, \lambda, G_{a}, R C S, B, F$ and $P_{\text {noise }}$ are fixed, thus the $S N R$ is a function in terms of the radar range $(R)$.

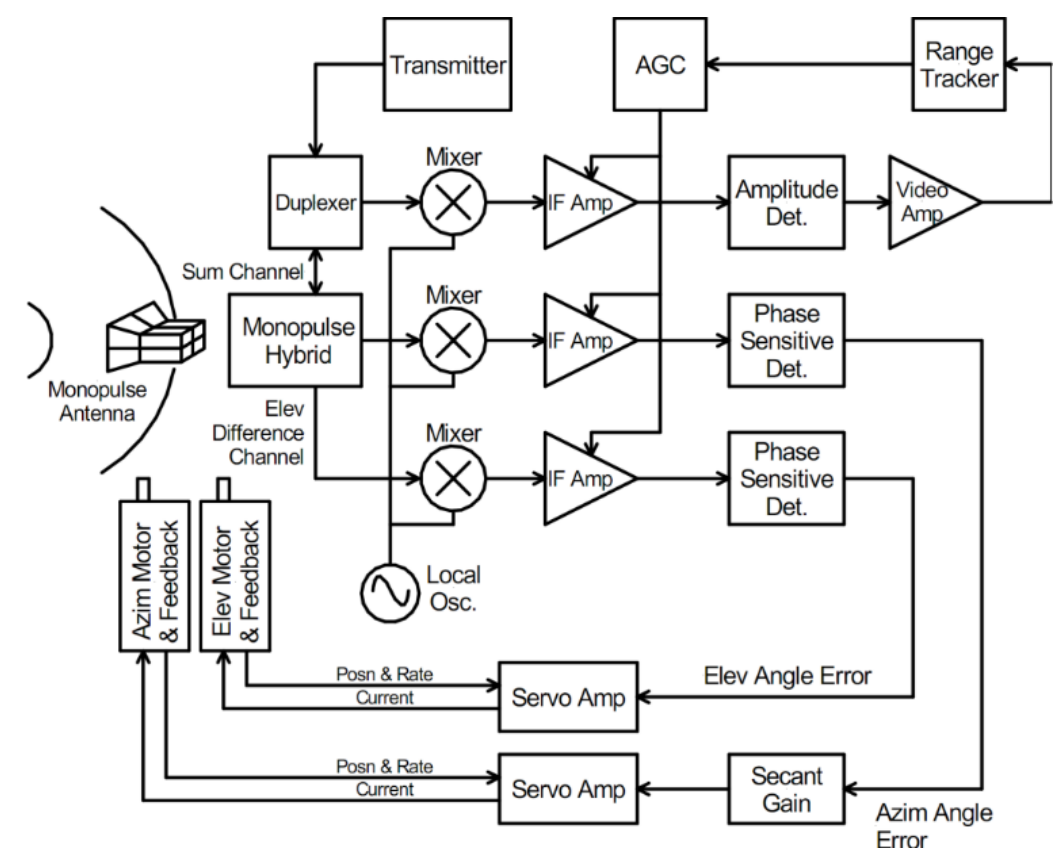

Figure 3. Block diagram of a monopulse tracking radar [2]

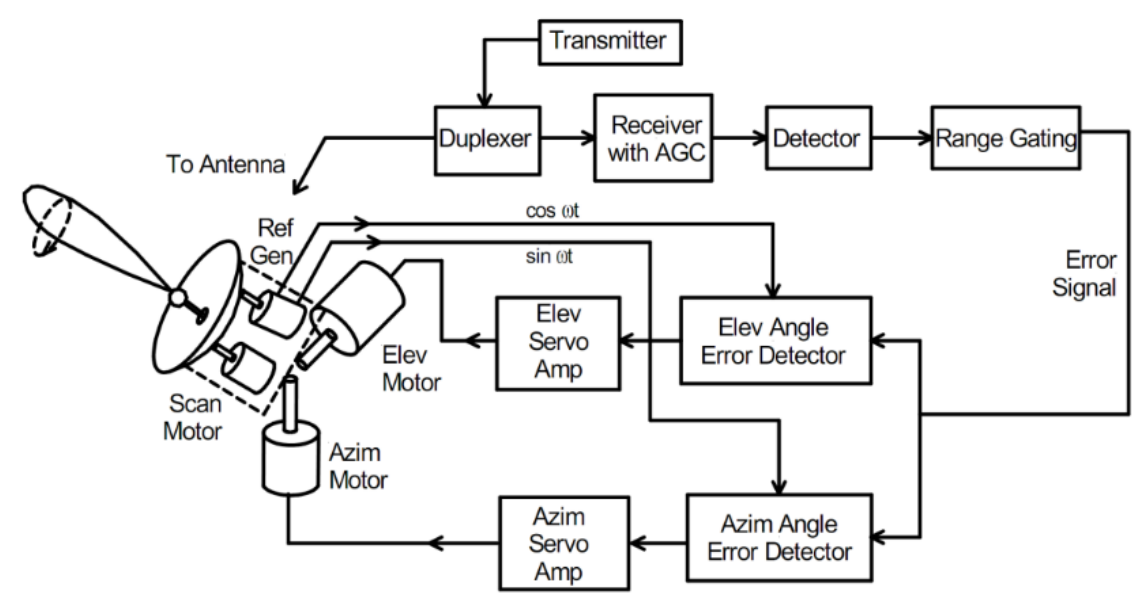

Figure 4. Block diagram of a conical scan radar [3]

\section{PROPOSED METHOD}

In this part, the proposed method is represented based on solving a mathematical optimization problem. In the previous part, we reviewed all details of angle error. It is noticeable that in an example with the specified parameters, $\sigma_{t}$ is only in terms of $R$ (distance to the target). Therefore, if we minimize the general standard deviation for a sample range such as $R=R_{\text {opt. }}$, then the total angle error becomes the minimum (because the mean of error variable is assumed to be zero, so a minimum variance/standard deviation shows the lowest error). In this case, $R_{\text {opt }}$ is the optimal range. The equation of $\sigma_{t}$ is rewritten as (10). If the SNR equation is to be rewritten as (11), we accordingly have $\sigma_{t}$ as (12). 


$$
\begin{aligned}
& \left.\left.\sigma_{t}=(\underbrace{\sigma_{\text {cont. }}^{2}+\sigma_{\text {mect. }}^{2}+\sigma_{\text {sc }}^{2}+\left(\frac{L}{3} \times \frac{1}{R}\right)^{2}+\left(\frac{\left(\frac{\theta_{B}}{q_{2}}\right)^{2}}{\sqrt{K_{4}}} \times \frac{1}{\left(\frac{2 P R F}{B_{s}}\right)}\right.}_{K_{1}})_{K_{2}}^{\frac{1}{S N R}}\right)\right)^{\frac{1}{2}} \\
& S N R=\underbrace{\frac{P_{t} \lambda^{2} G_{a}^{2} R C S}{(4 \pi)^{3} P_{\text {noise }}}}_{K_{3}} \times \frac{1}{R^{4}} \\
& \sigma_{t}=\left(K_{1}+\frac{K_{2}}{K_{3}} R^{4}+\frac{K_{4}}{R^{2}}\right)^{\frac{1}{2}}
\end{aligned}
$$

In (12), all $K_{1}$ to $K_{4}$ coefficients are fixed, so we find the optimal range which can minimize the term for $\sigma_{t}$, as (13). After solving the optimization problem, we see that $K_{1}$ has no impact on the $R_{\text {opt. }}$.

$$
\begin{gathered}
\frac{\partial \sigma_{t}}{\partial R}=0 \rightarrow A \cdot B=0 \\
\text { where } A=\left(K_{1}+\frac{K_{4}}{R^{2}}+\frac{K_{2}}{K_{3}} R^{4}\right)^{-\frac{1}{2}} \\
B=\frac{-2 K_{4}}{R^{3}}+\frac{K_{2}}{K_{3}}\left(4 R^{3}\right)
\end{gathered}
$$

The roots of (13) include all roots of two new equations of $A=0$ and $B=0$. It is provable that the equation $A=0$ has no acceptable root for $R$ (do it easily), we must solve the equation $B=0$; (14) shows the single root of this equation.

$$
R_{\text {opt. }}=\left(\frac{K_{3} K_{4}}{2 K_{2}}\right)^{\frac{1}{6}}
$$

The achieved relation for the optimal range is a theoretical solution. This recent equation is a generally closed form for all scanning techniques due to the consideration of the coefficient $q_{2}$ (embedded in $K_{2}$ ) and the standard deviation of $\sigma_{s c}$ in terms of different aspects related to the scanning techniques. In order to have a clear approach, we integrate all discussed items through the Consequence 1.

Consequence 1. The optimal range of an angle tracking radar for all scanning techniques is as (15)-(18).

$$
\begin{aligned}
& R_{\text {opt. }}=\sqrt[6]{a_{1} a_{2} a_{3}} \\
& a_{1}=\frac{P_{t} \lambda^{2} G_{a}^{2} R C S}{(4 \pi)^{3} P_{\text {noise }}} \\
& a_{2}=L^{2} / 9 \\
& a_{3}=\frac{K^{2} P R F}{\theta_{B}^{2} B_{s}} \\
& K \in\{1.3,1.7\} \quad\left(\equiv q_{2}\right)
\end{aligned}
$$


All angles are in degree(s) and other parameters are in linear space (not in $\mathrm{dB}$ ) and some of them which have physical dimension are in S.I. units. In the next part, we solve an example based on the proposed approach and then compare it to the simulation result.

\section{NUMERICAL EXAMPLE AND SIMULATION}

In this part, we firstly use the MATLAB code in the appendix to solve the problem by simulation (classically). Its result is shown in Figure 5. It is clear that the optimal range when the simulation parameters are selected as Table 1 is between 5 and 10 meters.

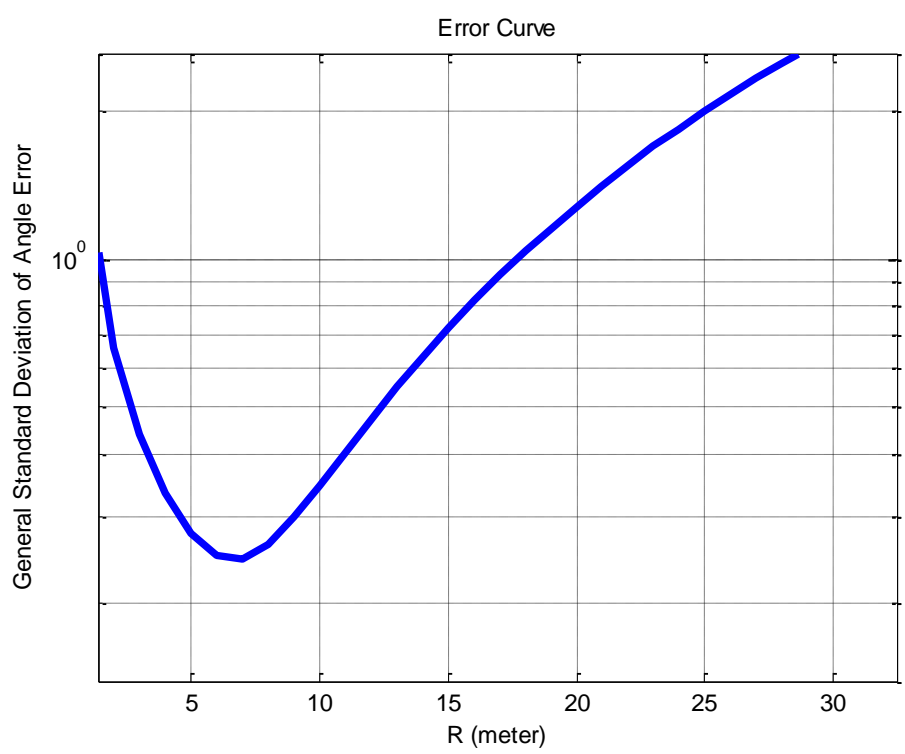

Figure 5. The error curve shows the optimal range obtained from the simulation under the setting in Table 1

Table 1. Settings for simulation

\begin{tabular}{l}
$\omega_{\max }=0.1 \mathrm{~m}$ \\
$k_{v}=10$ \\
$\sigma_{v}=\omega_{\max } / k_{v}=0.001$ \\
$\theta_{B}=2^{\circ}$ \\
$\sigma_{\text {mech } .}=0.002 \theta_{B}=0.004$ \\
$L=4 \mathrm{~m}$ \\
$\sigma_{g}=\frac{L}{3 R}=\frac{1.34}{R}$ \\
$B_{s}=30$ \\
$P R F=1000$ \\
$P_{t}=1000$ \\
$P_{\text {noise }}=1$ \\
$\lambda=0.03 \mathrm{~m}$ \\
$G_{a}=800$ \\
$R C S=12 \mathrm{~m}^{2}$ \\
$K=1.3$ \\
\hline
\end{tabular}


Now, we use the Consequence 1 for computing the optimal range theoretically. At first, $a_{1}, a_{2}$ and $a_{3}$ are computed by (16) to (18).

$$
\begin{aligned}
& a_{1}=3483.16 \\
& a_{2}=7.11 \\
& a_{3}=14.08
\end{aligned}
$$

Then with using (15), we calculate the optimal range as follow.

$$
\begin{aligned}
R_{\text {opt. }} & =\sqrt[6]{3483.16 \times 7.11 \times 14.08} \\
& =8.45 \mathrm{~m} \in[5,10]
\end{aligned}
$$

Note that in theoretical computation of the optimal range, some of the parameters like $\omega_{\max }, k_{v}$ and so on are not generally used (against the simulation-based solution, The MATLAB code for solving the problem based on simulation is in Table 2.).

\section{CONCLUSIONS}

In this work, our aim is to introduce a computational method [5]-[10] in which we theoretically computed the optimal range of ATRs. Our approach's result was matched with the simulation result in which they are sufficiently similar to each other. In fact, main aim of the work is to create an extension for the approaches represented in [1]. The proposed approach is suitable for educational aspects too.

\section{REFERENCES}

[1] M. Skolnik, "Introduction to Radar Systems," McGraw-Hill, 2001.

[2] G. Brooker, "Sensors and Signals: Tracking Moving Targets," Chap. 15, University of Sydney, 2006.

[3] G. Brooker, "Sensors and Signals: Range and Angle Tracking," Chap. 13, University of Sydney, 2006.

[4] S. Samadi, "Lecture Notes on Advanced Radar Systems," SUTech, 2017.

[5] M. Alhihi, M. R. Khosravi, H. Attar, M. Samour, "Determining the Optimum Number of Paths for Realization of Multi-path Routing in MPLS-TE Networks," Telecommunication. Computing Electronics and Control (TELKOMNIKA), vol. 15(4), pp. 1701-1709, 2017.

[6] M. Alhihi, M. R. Khosravi, "Operating Task Redistribution in Hyperconverged Networks," International Journal of Electrical and Computer Engineering (IJECE), vol. 8(3), pp. 1629-1635, 2018.

[7] P. K. Mane, K. N. Rao, "Granular Mobility-Factor Analysis Framework for enriching Occupancy Sensing with Doppler Radar," International Journal of Electrical and Computer Engineering (IJECE), vol. 8(2), pp. 979-988, 2018.

[8] M. Alhihi, M. R. Khosravi, "Formulizing the fuzzy rule for takagi-sugeno model in network traffic control," The Open Electrical and Electronic Engineering Journal, vol. 12(1), pp. 1-11, 2018.

[9] M. Ahmed, M. Salleh, M. I. Channa, M. F. Rohani, "Review on Localization based Routing Protocols for Underwater Wireless Sensor Network," International Journal of Electrical and Computer Engineering (IJECE), vol. 7(1), pp. 536-541, 2017.

[10] M. Ahmed, M. Salleh, M. I. Channa, M. F. Rohani, "RMEER: Reliable Multi-path Energy Efficient Routing Protocol for Underwater Wireless Sensor Network," International Journal of Electrical and Computer Engineering (IJECE), vol.8(6), pp. 4366-4373, 2018. 
APPENDIX

Table 2. The MATLAB code for solving the problem based on simulation sigma_t $=$ zeros $(1,1000)$;

for $\mathrm{R}=1: 1: 1000$

$\mathrm{W} \_\max =0.01$

$\mathrm{k} \_\mathrm{v}=10$;

sigma_v2 $=\left(\mathrm{w}_{-} \mathrm{max} / \mathrm{k} \_\mathrm{v}\right) \mathbf{.}^{\wedge} 2$;

theta_b $=2 ; \%$ azimuth/elevation beam width

sigma_mec $2=\left(0.002^{*} \text { theta_b }\right)^{\wedge} 2$;

$\mathrm{L}=4 ; \%$ it is the largest dimension of the tracked target.

sigma_g2 $=\left(0.33^{*}(\mathrm{~L} / \mathrm{R})\right)$. $^{\wedge}$;

$\mathrm{Bs}=30$;

$\mathrm{PRF}=1000$;

$\mathrm{Pt}=1000$

Lambda $=0.03$;

$\mathrm{Ga}=800$;

$\mathrm{RCS}=12$

$\mathrm{SNR}=\left(\mathrm{Pt}^{*}\left(\mathrm{Lambda} .{ }^{\wedge} 2\right)^{*}\left(\mathrm{Ga} .^{\wedge} 2\right)^{*} \mathrm{RCS}\right) /\left(\left(64^{*}\left(\mathrm{pi}^{\wedge} 3\right)\right)^{*}\left(\mathrm{R} .{ }^{\wedge} 4\right)\right)$;

$\%$ assume Pnoise $=1$

sigma_n2 $=\left(\right.$ theta_b/ $\left.\left(1.3^{*} \operatorname{sqrt}\left(2^{*} \mathrm{SNR}^{*}(\mathrm{PRF} / \mathrm{Bs})\right)\right)\right) \cdot{ }^{\wedge} 2$;

sigma_sc2 $=\left(0.01^{*}\right.$ theta_b $) .2$;

sigma_t(floor $(\mathrm{R}))=$ sqrt $($ sigma_v2 +sigma_mec2 + sigma_g2

+ sigma_n2 + sigma_sc2);

end

figure;

$\mathrm{RR}=1: 1: 1000$;

$\log \log ($ RR,sigma_t $)$;grid;

axis([0 $10010^{\wedge}(-1)$ 100]);

title('Error Curve');

xlabel('R (meter)');

ylabel('General Standard Deviation of Angle Error'); 